\title{
Physical Exercise Improves Heart-Rate Variability in Obese Children and Adolescents: A Systematic Review
}

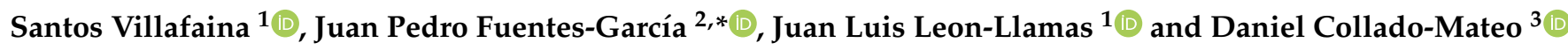 \\ 1 Physical Activity and Quality of Life Research Group (AFYCAV), Faculty of Sport Sciences, \\ University of Extremadura, 10004 Cáceres, Spain; svillafaina@unex.es (S.V.); leonllamas@unex.es (J.L.L.-L.) \\ 2 Faculty of Sport Science, University of Extremadura, Avda: Universidad S/N, 10003 Cáceres, Spain \\ 3 Centre for Sport Studies, Rey Juan Carlos University, 28943 Fuenlabrada, Spain; danicolladom@gmail.com \\ * Correspondence: jpfuent@unex.es
}

Citation: Villafaina, S.;

Fuentes-García, J.P.; Leon-Llamas, J.L.; Collado-Mateo, D. Physical Exercise Improves Heart-Rate Variability in Obese Children and Adolescents: A Systematic Review. Sustainability 2021, 13, 2946. https://doi.org/ $10.3390 /$ su13052946

Academic Editor: Giuseppe Battaglia

Received: 3 February 2021

Accepted: 3 March 2021

Published: 8 March 2021

Publisher's Note: MDPI stays neutral with regard to jurisdictional claims in published maps and institutional affiliations.

Copyright: (c) 2021 by the authors. Licensee MDPI, Basel, Switzerland. This article is an open access article distributed under the terms and conditions of the Creative Commons Attribution (CC BY) license (https:// creativecommons.org/licenses/by/ $4.0 /)$.

\begin{abstract}
Background: Childhood obesity has negative impact on heart-rate variability (HRV) and, thereby, on the cardiovascular health of children and adolescents. Thus, physical-exercise interventions were proposed to increase HRV. The present systematic review aims to provide an up-to-date analysis of research on the effect of physical-exercise interventions on HRV in obese children and adolescents. Methods: An electronic search of the literature was performed, and 10 articles were included. PRISMA guideline methodology was employed. Results: Physical-exercise interventions predominantly involved aerobic training; however, alternative training programs, including judo or recreational soccer, were found. The duration of intervention ranged from 6 to 24 weeks, with a training frequency of between 2 and 7 times per week. The duration of sessions typically ranged from 40 to $60 \mathrm{~min}$. Conclusions: Results of the included articles indicated that physical-exercise intervention increased the HRV and thereby the autonomic modulation of obese children and adolescents. This is significant, as HRV is associated with cardiovascular health. Such physical-exercise interventions are crucial to reduce weight and improve cardiovascular health in children and adolescents, thereby achieving a sustainable future.
\end{abstract}

Keywords: HRV; autonomic modulation; physical activity

\section{Introduction}

Global obesity prevalence among children has increased in recent decades. It is estimated that 124 million children and adolescents between 5 and 19 years of age are obese, and more than 213 million are overweight [1]. Obesity has enormous health consequences. For instance, obesity during childhood increases cardiovascular risk [2,3] and cognitive decline in terms of executive function [4]. Furthermore, childhood obesity has enormous economic impact [5]. In this regard, one goal of sustainable development is to ensure healthy lives and promote wellbeing at all ages [6]. Since childhood obesity dramatically compromises health, physical-exercise interventions are crucial to reduce weight, improve cardiovascular health in children and adolescents, and achieve a sustainable future [7-9].

The World Health Organization recommends performing at least $60 \mathrm{~min}$ of vigorous physical activity per day, and strengthening muscle and bone through games, jumps, and sprints in order to counteract obesity [10]. As a result, aerobic exercise emerged as a relevant component of physical-activity intervention to reduce obesity. Such training can effectively decrease body fat [11], improve cardiorespiratory fitness [12,13], or reduce cardiovascular risk factors [14-16]. Despite the benefits of physical activity, Guthold, Stevens [17] revealed that the overall prevalence of insufficient physical activity among adolescents is higher than $80 \%$.

Heart-rate variability (HRV) is a noninvasive measure of the autonomic nervous system that is based on the study of successive RR intervals. This measure provides 
information about the balance between the sympathetic and parasympathetic nervous systems [18]. Low HRV is associated with increased risk of mortality from several causes [19] and increased obesity status or central adiposity [20]. Furthermore, obese adolescents show cardiac autonomic dysfunction (indicated by reduced HRV), which leads to poor cardiovascular health and decreased parasympathetic function [21,22]. However, higher HRV values are typically present in individuals with high fitness levels or after exercise [23]. In this regard, physical activity such as aerobic training [24], recreational soccer [25], highintensity training [26], or judo [27] are used as interventions to increase HRV and thereby decrease sympathetic modulation.

To our knowledge, neither systematic reviews nor meta-analyses have explored the effect of physical-exercise interventions on HRV in obese adolescents and children. Therefore, the present review provides up-to-date analysis of studies published in scientific journals that are indexed in well-known databases on the effects of physical-exercise interventions on HRV in obese children and adolescents, and offers future directions.

\section{Materials and Methods}

This systematic review was conducted following the PRISMA guidelines [28].

\subsection{Data Sources and Searches}

Two well-known databases, PubMed and Web of Science (WOS) (including Current contents connect, Derwent innovations index, Korean journal database, Medline, Russian science citation index and, SciELO citation index), were used to extract eligible articles. The used search term string was: (child* OR adolescen* OR kid) AND (obes* OR overweight) AND (exer* OR "physical therapy") AND ("heart rate variability" OR HRV OR "autonomic modulation").

The article selection process is shown in Figure 1. The search was conducted by SV and supervised by JLLL. In cases of disagreement, DCM directed a consensus discussion. The search ended on 5 January 2021, and only articles published after 1970 were potentially included.

Studies were included if they met the following inclusion criteria: (a) quantitative randomized controlled trial or observational designs focused on exercise interventions, (b) samples comprising adolescents or children, (c) adolescents or children suffering from obesity, and (d) the article reported HRV outcomes. Furthermore, articles that met any of the following criteria were excluded: (a) studies not written in Spanish, Portuguese, English, Italian, or French; (b) summaries of conferences or seminars; and (c) commentaries, editor letters, dissertations, or theses.

\subsection{Risk of Bias}

The Evidence Project risk of bias tool [29] was used to assess the risk of bias in the selected articles. Eight criteria were assessed as: yes, no, not applicable, or not reported. These criteria were: (1) cohort, (2) control or comparison group, (3) pre-/postintervention data, (4) random assignment of participants to the intervention, (5) random selection of participants for assessment, (6) follow-up rate of $80 \%$ or more, (7) comparison groups equivalent on sociodemographics, and (8) comparison groups equivalent at baseline on outcome measures. This information is summarized in Table 1. 

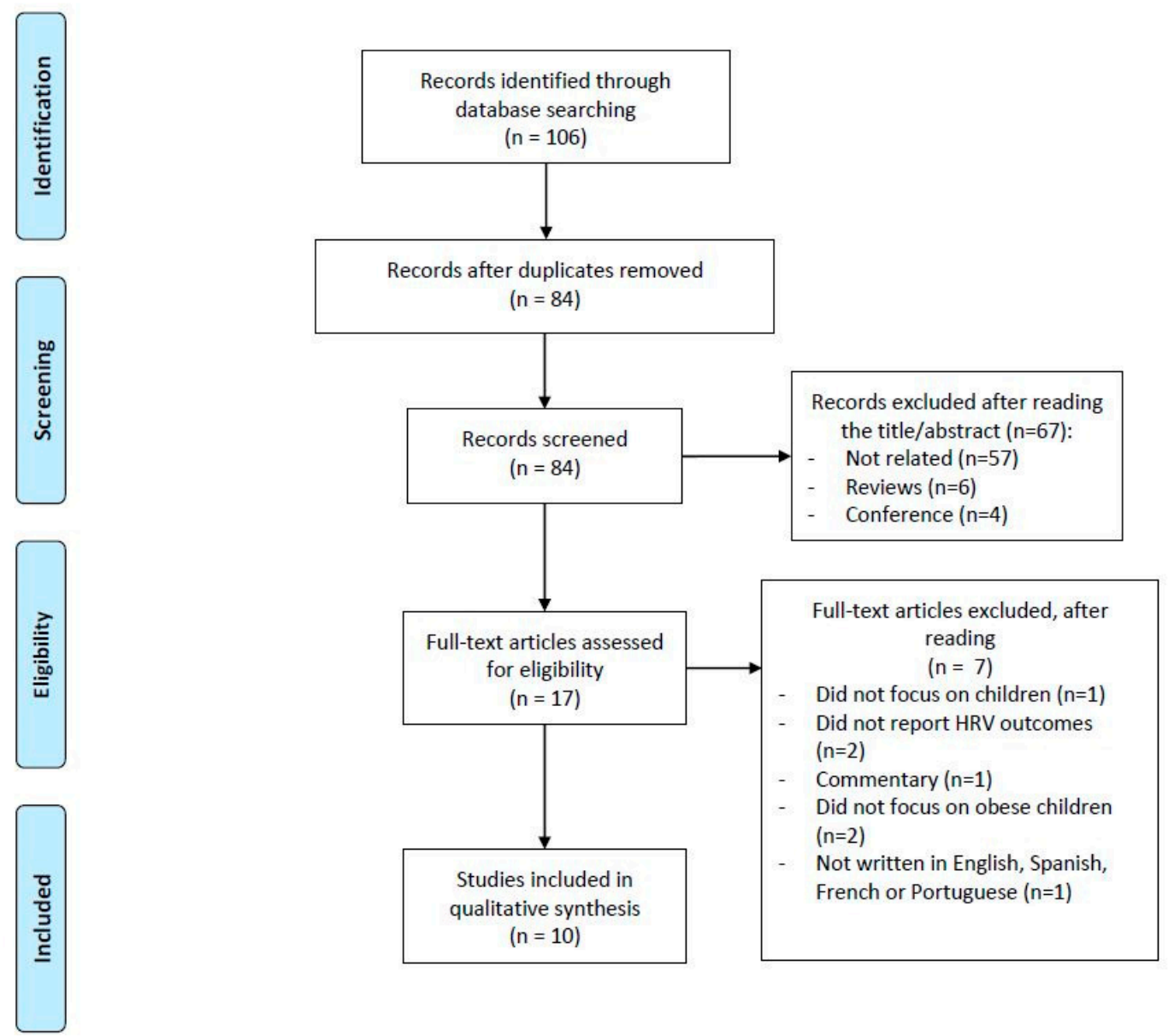

Figure 1. Article selection process flow diagram.

\subsection{Data Extraction}

The Participants, Intervention, Comparison, Outcome, and Study (PICOS) design strategy, as recommended by the PRISMA methodology, was used to extract data from articles [28]. This information is summarized in Tables $2-4$. 
Table 1. Risk of bias in randomized and nonrandomized controlled trials using Evidence Project risk of bias tool.

\begin{tabular}{|c|c|c|c|c|c|c|c|c|}
\hline Study & Cohort & $\begin{array}{l}\text { Control or } \\
\text { Comparison } \\
\text { Group }\end{array}$ & $\begin{array}{c}\text { Pre- } \\
\text { /Postintervention } \\
\text { Data }\end{array}$ & $\begin{array}{c}\text { Random } \\
\text { Assignment of } \\
\text { Participants to } \\
\text { Intervention }\end{array}$ & $\begin{array}{l}\text { Random } \\
\text { Selection of } \\
\text { Participants for } \\
\text { Assessment }\end{array}$ & $\begin{array}{c}\text { Follow-Up } \\
\text { Rate of } 80 \% \\
\text { or More }\end{array}$ & $\begin{array}{c}\text { Comparison } \\
\text { Groups } \\
\text { Equivalent on } \\
\text { Sociodemographics }\end{array}$ & $\begin{array}{l}\text { Comparison } \\
\text { Groups } \\
\text { Equivalent } \\
\text { at Baseline on } \\
\text { Disclosure }\end{array}$ \\
\hline \multicolumn{9}{|l|}{ Brasil (2020) } \\
\hline \multicolumn{9}{|l|}{ Chen (2016) } \\
\hline \multicolumn{9}{|l|}{ Farah (2012) } \\
\hline \multicolumn{9}{|l|}{ Farinatti (2016) } \\
\hline \multicolumn{9}{|l|}{ Faulkner (2013) } \\
\hline \multicolumn{9}{|l|}{ Gutin (2000) } \\
\hline \multicolumn{9}{|l|}{ Hamila (2017) } \\
\hline \multicolumn{9}{|l|}{ Huang (2019) } \\
\hline Vasconcellos (2015) & & & & & & & & \\
\hline
\end{tabular}




\section{Results}

\subsection{Article Selection}

The article selection process is depicted in Figure 1. A total of 106 articles were identified in electronic databases WOS (65 articles) and PubMed (41 articles). Twenty-two articles were duplicates. Furthermore, 67 articles were excluded after reading the title or abstract (reasons detailed in Figure 1). Of the remaining 17 articles, 7 were excluded as per the exclusion criteria. Therefore, 10 articles were included in the qualitative synthesis.

\subsection{Risk of Bias}

Table 1 shows the results of the risk of bias assessment. Random assignment of participants to intervention ( $40 \%$ of articles did not fulfill this criterion) [27,30-32], random selection of participants for assessment ( $0 \%$ of articles fulfilled this criterion), and follow-up rate of $80 \%$ or more (30\% of articles did not fulfill this criterion) $[26,27,31]$ were the most important sources of bias detected in the selected articles.

\subsection{Participants}

Table 2 displays the participants' characteristics in each study. Three articles were focused on obese children [27,33,34], whereas 7 were focused on obese adolescents $[25,26,30-32,35,36]$. Sample sizes ranged between 7 and 79 participants per study, a total of 335 participants (49.25\% girls). Participants' ages ranged from 9 to 15 years.

Table 2. Characteristics of samples and study designs of included articles.

\begin{tabular}{|c|c|c|c|c|c|}
\hline Study (Year) & Participants & $\begin{array}{l}\text { Sample } \\
\text { Size (N) }\end{array}$ & Age (SD) & Study Design & Control Group Protocol \\
\hline Brasil (2020) & $\begin{array}{c}\text { Obese children } \\
\text { Nonobese children }\end{array}$ & $\begin{array}{l}20(10 \mathrm{~g}) \\
15(7 \mathrm{~g})\end{array}$ & $\begin{array}{l}11.1(1.1) \\
10.7(1.6)\end{array}$ & Non-RCT & Usual life \\
\hline Chen (2016) & Obese adolescents & $\begin{array}{l}25(13 \mathrm{~g}) \\
25(9 \mathrm{~g})\end{array}$ & $\begin{array}{l}\text { EG: } 12.64(0.70) \\
\text { CG: } 12.84(0.75)\end{array}$ & $\mathrm{RCT}$ & Usual life \\
\hline Farah (2012) & Obese adolescents & $\begin{array}{c}9(5 \mathrm{~g}) \\
10(5 \mathrm{~g})\end{array}$ & $\begin{array}{l}\text { HIT: } 15.4(0.4) \\
\text { LIT: } 14.8(0.4)\end{array}$ & $\mathrm{RCT}$ & Light-intensity training \\
\hline Farinatti (2016) & $\begin{array}{c}\text { Obese adolescents } \\
\text { Nonobese adolescents }\end{array}$ & $\begin{array}{l}24(17 \mathrm{~g}) \\
20(7 \mathrm{~g})\end{array}$ & $13-17$ & Non-RCT & Usual life \\
\hline Faulkner (2013) & $\begin{array}{c}\text { Obese adolescents } \\
\text { Type II DM adolescents }\end{array}$ & $\begin{array}{l}10(6 \mathrm{~g}) \\
9(8 \mathrm{~g})\end{array}$ & $\begin{array}{l}14.6(1.6) \\
14.7(1.8)\end{array}$ & Non-RCT & Personalized aerobic training \\
\hline Gutin (2000) & Obese children & $79(53 \mathrm{~g})$ & $9.5(1)$ & Crossover & Usual life \\
\hline Hamila (2017) & Obese adolescents & $\begin{array}{l}7(4 \mathrm{~g}) \\
8(5 \mathrm{~g})\end{array}$ & $\begin{array}{l}\text { EG: } 14.5(1) \\
\text { CG: } 14.5(0.9)\end{array}$ & $\mathrm{RCT}$ & Usual life \\
\hline Huang (2019) & Obese adolescents & $21(10 \mathrm{~g})$ & $10-16$ & Observational & - \\
\hline Prado (2010) & Obese children & $\begin{array}{l}18 \\
15\end{array}$ & $\begin{array}{l}\text { EG: } 10.3(0.2) \\
\text { CG: } 10.2(0.3)\end{array}$ & $\mathrm{RCT}$ & Diet \\
\hline Vasconcellos (2015) & Obese adolescents & $\begin{array}{l}10(4 \mathrm{~g}) \\
10(2 \mathrm{~g})\end{array}$ & $\begin{array}{l}\text { EG: } 14.3(1.3) \\
\text { CG: } 14.8(1.4)\end{array}$ & $\mathrm{RCT}$ & Usual life \\
\hline
\end{tabular}

g, girls; DM, diabetes mellitus; RCT, randomized controlled trial; EG, experimental group; CG, control group; HIT, high-intensity training; LIT, light-intensity training; N, sample size; SD, standard deviation.

\subsection{Study Design}

Table 2 presents the study design. Five (50\% of total) articles were classified as randomized controlled trials [25,26,34-36]. Three articles were classified as nonrandomized controlled trials $[27,30,31]$ (two groups with pre- and postassessments but without randomization). Furthermore, one article had a crossover design [33], whereas another had an observational design [32] (the experimental group was only assessed once at the end of the intervention).

\subsection{Intervention}

Table 3 summarizes the intervention protocols of the treatment groups in each study. All interventions included an experimental group comprising participants who performed exercise. The duration of interventions ranged from 6 to 24 weeks with a training frequency of between 2 and 7 times per week. Session durations typically ranged between 40 and $60 \mathrm{~min}$ except for one intervention that required a total of $5 \mathrm{~h} /$ day of physical activity [32]. 
Table 3. Frequency, duration, and intensity of physical-exercise interventions included in this systematic review.

\begin{tabular}{|c|c|c|c|c|c|}
\hline Study (Year) & $\begin{array}{l}\text { Intervention } \\
\text { Duration } \\
\text { (Weeks) }\end{array}$ & $\begin{array}{l}\text { Session } \\
\text { Duration } \\
\text { (Minute) }\end{array}$ & $\begin{array}{l}\text { Weekly } \\
\text { Frequency } \\
\text { (Days) }\end{array}$ & Intensity & Activities Included in Session \\
\hline Brasil (2020) & 12 & 60 & 2 & $\begin{array}{l}65-75 \% \text { maximal } \\
\text { heart rate }\end{array}$ & $\begin{array}{l}\text { Sessions consisted of } 60 \text { min of judo training for } \\
\text { beginners (including } 10 \text { min warmup and } \\
10 \text { min cooldown). }\end{array}$ \\
\hline Chen (2016) & 12 & 40 & 4 & $\begin{array}{c}60-70 \% \text { maximal } \\
\text { heart rate }\end{array}$ & $\begin{array}{l}\text { Participants were free to choose one of the provided } \\
\text { exercise types (e.g., fast walking, stair climbing, } \\
\text { jumping rope, or aerobic dancing). }\end{array}$ \\
\hline Farah (2012) & 24 & Not fixed & 31 & $\begin{array}{l}\text { HIT: at ventilatory } \\
\text { threshold I. } \\
\text { LIT: } 20 \% \text { below } \\
\text { ventilatory } \\
\text { threshold I. }\end{array}$ & Treadmill. \\
\hline Farinatti (2016) & 12 & $30-40$ & 3 & $\begin{array}{c}50-85 \% 10 \mathrm{RM}, \\
\text { progressively }\end{array}$ & $\begin{array}{l}\text { One set of } 10-15 \text { repetitions (reps) with } 50-70 \% \text { of load } \\
\text { corresponding to } 10 \text { RM for first } 2 \text { weeks; two sets of } \\
8-12 \text { reps with } 60-80 \% 10 \text { RM in weeks } 3-6 \text {, and three } \\
\text { sets of } 6-10 \text { reps with } 70-85 \% 10 \text { RM in weeks } 7-12 \text {. }\end{array}$ \\
\hline Faulkner (2013) & 16 & 60 & 7 & $\begin{array}{c}65-75 \% \text { maximal } \\
\text { heart rate }\end{array}$ & $\begin{array}{l}\text { Personalized training (based on participant interest) } \\
\text { where participants performed activities such as } \\
\text { calisthenics, kickboxing, dancing, cycling, walking, } \\
\text { and Dance Dance Revolution (Konami, Japan). } \\
\text { Activities could be conducted at gym facilities, parks, } \\
\text { schools, participants' homes, or all of the above. }\end{array}$ \\
\hline Gutin (2000) & 16 & 40 & 5 & - & $\begin{array}{l}\text { First } 20 \text { min were spent on machines (e.g., treadmill, } \\
\text { cycle, Nordic ski machine), and next } 20 \text { min were } \\
\text { devoted to games modified to maintain a high rate of } \\
\text { energy expenditure. }\end{array}$ \\
\hline Hamila (2017) & 8 & 50 & 3 & $\begin{array}{l}70 \% \text { maximal } \\
\text { aerobic speed }\end{array}$ & $\begin{array}{l}\text { Each session included a } 10 \text { min collective warmup } \\
\text { based on ball games followed by } 2 \times 20 \text { min periods of } \\
\text { walking, interspersed by } 10 \text { maximal sprints on a cycle } \\
\text { ergometer against a braking force equal to } 0.75 \mathrm{~g} / \mathrm{kg} \\
\text { body mass. }\end{array}$ \\
\hline Huang (2019) & 6 & $5 \mathrm{~h} /$ day & 6 & - & $\begin{array}{l}\text { Program primarily comprised various types of aerobic } \\
\text { exercise such as bicycling, walking, running, } \\
\text { dancing, and ball games for } 5 \mathrm{~h} / \text { day. It was } \\
\text { supplemented by strength training. Endurance } \\
\text { exercises involved moderate- ( } 70-85 \% \text { of maximal } \\
\text { heart rate) and high-intensity ( } 90 \% \text { of maximal heart } \\
\text { rate) training. } \\
\text { Strength training was conducted } 2-3 \text { times per week at } \\
40-50 \% \text { maximal strength for } 2-3 \text { sets of } 12-15 \\
\text { repetitions maximum, with } 2-3 \text { min of rest between } \\
\text { sets. Furthermore, participants were provided with } \\
\text { calorie-restricted but nutritionally complete diet based } \\
\text { on their age. }\end{array}$ \\
\hline Prado (2010) & 16 & 60 & 3 & $\begin{array}{l}\text { Ventilatory } \\
\text { anaerobic } \\
\text { threshold }\end{array}$ & $\begin{array}{c}\text { Each exercise session consisted of } 30 \text { min of walking } \\
\text { and / or jogging (aerobic exercise) on a jogging track, } \\
\text { and } 30 \text { min of recreational exercise. }\end{array}$ \\
\hline $\begin{array}{c}\text { Vasconcellos } \\
\text { (2015) }\end{array}$ & 12 & 60 & 3 & - & $\begin{array}{c}\text { Each session consisted of a } 10 \text { min warmup followed } \\
\text { by } 40 \text { min of games performed in small pitch areas } \\
\text { (such as } 2 \text { vs. } 2,3 \text { vs. } 3 \text {, and } 4 \text { vs. 4), and a } \\
10 \text { min cooldown. }\end{array}$ \\
\hline
\end{tabular}

A total of 6 articles conducted aerobic training in the range of $60-75 \%$ of maximal heart rate $[27,31,35]$, at the first ventilatory threshold [26], at $70 \%$ of the maximal aerobic speed [36], or at the ventilatory anaerobic threshold [34]. One article conducted resistance training at 50-85\% 10 repetition maximum (RM) [30]. The three remaining articles did not report intensity; however, their interventions were based on aerobic training [33], aerobic plus resistance training [32], and recreational soccer [25]. 


\subsection{Comparison Groups}

Table 2 provides the protocol followed by the comparison groups. Two articles included a nonobese adolescent group [27,30], 1 Type II diabetes mellitus group [31], 1 did not include a comparison group [32], and the 6 other articles included obese children or adolescents as a comparison group [25,26,33-36].

Among studies with a control group, 6 articles encouraged the control group to follow their usual life $[25,27,30,33,35,36]$. In contrast, the control group of one study performed light-intensity training [26], that of another article performed personalized aerobic training [31], and a third control group followed a diet (see Table 2) [34].

\subsection{Outcomes}

HRV results from the selected articles are summarized in Table 4 . Time- and frequencydomain variables were the most studied variables. Eight articles reported within- or between-group differences after physical training on HRV variables. Only 1 article did not report any significant differences [31].

Table 4. Results analysis of selected articles.

\begin{tabular}{|c|c|c|c|c|c|c|c|}
\hline Authors & $\begin{array}{l}\text { Recording Protocol } \\
\text { and Instrument }\end{array}$ & $\begin{array}{l}\text { Outcome } \\
\text { Measure }\end{array}$ & EG Baseline & $\begin{array}{l}\text { EG after } \\
\text { Treatment }\end{array}$ & CG Baseline & $\begin{array}{l}\text { CG after } \\
\text { Treatment }\end{array}$ & $\begin{array}{c}\text { Reported } \\
\text { Effect }\end{array}$ \\
\hline \multirow{7}{*}{ Brasil (2020) } & \multirow{7}{*}{$\begin{array}{c}5 \mathrm{~min} \\
\text { (Polar RS800cx, }^{\mathrm{TM}} \\
\text { Polar }^{\mathrm{TM}}, \text { Kempele, } \\
\text { Finland) }\end{array}$} & $\mathrm{RR}(\ln \mathrm{ms})$ & $2.85(0.02)$ & $2.88(0.02)$ & $2.88(0.02)$ & - & WG \\
\hline & & SDNN (ln ms) & $1.66(0.06)$ & $1.64(0.07)$ & $1.66(0.05)$ & - & - \\
\hline & & Rmssd (ln ms) & $1.64(0.07)$ & $1.63(0.07)$ & $1.67(0.07)$ & - & - \\
\hline & & Pnn50 (ln \%) & $1.16(0.12)$ & $1.19(0.11)$ & $1.24(0.12)$ & - & - \\
\hline & & $\mathrm{LF}\left(\ln \mathrm{ms}^{2}\right)$ & $1.75(0.03)$ & $1.72(0.02)$ & $1.71(0.02)$ & - & - \\
\hline & & $\mathrm{HF}\left(\ln \mathrm{ms}^{2}\right)$ & $1.63(0.02)$ & $1.70(0.03)$ & $1.66(0.03)$ & - & WG \\
\hline & & $\mathrm{LF} / \mathrm{HF}\left(\ln \mathrm{ms}^{2}\right)$ & $0.13(0.05)$ & $0.02(0.03)$ & $0.06(0.04)$ & - & BG (pre)/WG \\
\hline \multirow[t]{2}{*}{ Chen (2016) } & \multirow{2}{*}{$\begin{array}{c}5 \mathrm{~min} \\
\text { Handheld device } \\
\text { (CheckMyHeart 3.0, } \\
\text { DailyCare } \\
\text { BioMedical, Inc., } \\
\text { Taoyuan, Taiwan) }\end{array}$} & $\operatorname{Lf}(n u)$ & $57.82(15.64)$ & $62.85(18.27)$ & $52.09(16.3)$ & $48.56(15.46)$ & BG \\
\hline & & Hf (nu) & 42.27 (13.38) & 42.37 (13.38) & 42.27 (13.38) & 42.37 (13.38) & EG (WG)/BG \\
\hline \multirow{6}{*}{ Farah (2012) } & \multirow{6}{*}{$\begin{array}{c}7 \mathrm{~min} \\
\text { (Polar RS800cx, } \\
\text { Polar }^{\mathrm{TM}}, \text { Kempele, } \\
\text { Finland) }\end{array}$} & Mean RR (ms) & $757(55)$ & - & $810(31)$ & - & $\mathrm{EG}(\mathrm{WG}) / \mathrm{BG}$ \\
\hline & & SDNN (ms) & $75(16)$ & - & $88(9)$ & - & \\
\hline & & rMSSD (ms) & $57(18)$ & - & $66(9)$ & - & \\
\hline & & Pnn50 (\%) & $26(10)$ & - & $33(5)$ & - & $\mathrm{EG}(\mathrm{WG}) / \mathrm{BG}$ \\
\hline & & $\mathrm{LF}\left(\mathrm{ms}^{2}\right)$ & 3941 (1320) & - & $5090(1000)$ & - & \\
\hline & & $\mathrm{HF}\left(\mathrm{ms}^{2}\right)$ & 1602 (1014) & - & $1842(392)$ & - & EG (WG) \\
\hline \multirow{8}{*}{ Farinatti (2016) } & \multirow{8}{*}{$\begin{array}{l}15 \mathrm{~min} \\
\text { Noninvasive device } \\
\text { (Finometer, Finapres } \\
\text { Medical Systems, } \\
\text { Amsterdam, The } \\
\text { Netherlands) }\end{array}$} & Mean RR (ms) & 746.2 (71.4) & - & $862.4(126)$ & - & WG \\
\hline & & SDNN (ms) & $58.3(25.5)$ & - & $79.8(35.9)$ & - & WG \\
\hline & & rMSSD (ms) & $46(18.4)$ & - & $74.3(21.5)$ & - & BG (pre)/WG \\
\hline & & Pnn50 (\%) & $24.8(16)$ & - & $44.6(13.6)$ & - & BG (pre)/WG \\
\hline & & LF (nu) & $50.4(16.3)$ & - & 31.4 (17.2) & - & \\
\hline & & Total power & - & - & - & - & WG \\
\hline & & HF (nu) & $49.6(16.3)$ & - & $68.6(17.2)$ & - & BG (pre)/WG \\
\hline & & $\mathrm{LH} / \mathrm{HF}$ ratio & $1.3(0.9)$ & - & $0.59(0.6)$ & - & BG (pre) \\
\hline
\end{tabular}


Table 4. Cont.

\begin{tabular}{|c|c|c|c|c|c|c|c|}
\hline Authors & $\begin{array}{l}\text { Recording Protocol } \\
\text { and Instrument }\end{array}$ & $\begin{array}{l}\text { Outcome } \\
\text { Measure }\end{array}$ & EG Baseline & $\begin{array}{c}\text { EG after } \\
\text { Treatment }\end{array}$ & CG Baseline & $\begin{array}{c}\text { CG after } \\
\text { Treatment }\end{array}$ & $\begin{array}{c}\text { Reported } \\
\text { Effect }\end{array}$ \\
\hline \multirow{7}{*}{ Faulkner (2013) } & \multirow{7}{*}{$\begin{array}{c}24 \mathrm{~h} \\
\text { Vision Premier } \\
\text { Holter Analysis } \\
\text { System Software, } \\
\text { (Cardiac Science, } \\
\text { Bothell, WA). }\end{array}$} & $\begin{array}{l}\text { Total Power } \\
\left(\ln \mathrm{ms}^{2}\right)\end{array}$ & $8.3(0.9)$ & $8.0(1)$ & $7.6(0.6)$ & $7.6(0.8)$ & - \\
\hline & & $\mathrm{HF}\left(\ln \mathrm{ms}^{2}\right)$ & $6.8(1)$ & $6.5(1.1)$ & $6(0.8)$ & $6.1(0.8)$ & - \\
\hline & & $\mathrm{LF}\left(\ln \mathrm{ms}^{2}\right)$ & $7(1)$ & $6.7(1.1)$ & $6.4(0.5)$ & $6.3(0.6)$ & - \\
\hline & & SDNN (ms) & $150(46)$ & $148(57)$ & $108(22)$ & $110(35)$ & - \\
\hline & & SDANN (ms) & $127(37)$ & $127(52)$ & $94(22)$ & $95(32)$ & - \\
\hline & & Pnn50 (\%) & $23(15)$ & $19(12)$ & $11(6)$ & $12(9)$ & - \\
\hline & & rMSSD (ms) & $72(37)$ & $62(36)$ & $41(15)$ & $46(25)$ & - \\
\hline Gutin (2000) & $\begin{array}{c}10 \text { min } \\
\text { Schiller ECG } \\
\text { system(Baar, } \\
\text { Switzerland) }\end{array}$ & rMSSD (ms) & $54.3(26.3)$ & $\begin{array}{c}\text { Change }=6.1 \\
(27.8)\end{array}$ & - & - & $\mathrm{BG}$ \\
\hline \multirow{9}{*}{ Hamila (2017) } & \multirow{9}{*}{$\begin{array}{c}5 \mathrm{~min} \\
\text { (Polar S-810, Polar }^{\mathrm{TM}} \text {, } \\
\text { Kempele, Finland) }\end{array}$} & Mean RR (ms) & $644(40.6)$ & $670.4(64.1)$ & $611(30.5)$ & $621.7(30.5)$ & - \\
\hline & & ln rMSSD & $1.58(0.2)$ & $1.78(0.2)$ & $1.53(0.19)$ & $1.62(0.33)$ & - \\
\hline & & $\mathrm{HF}(\mathrm{nu})$ & $24.6(14.4)$ & $34.5(15.1)$ & $27.02(8.98)$ & $28.1(6.46)$ & EG (WG) \\
\hline & & $\mathrm{HF}\left(\ln \mathrm{ms}^{2}\right)$ & $2.5(0.5)$ & $2.6(0.5)$ & $2.3(0.51)$ & $2.37(0.81)$ & - \\
\hline & & LF (nu) & $72.2(12.8)$ & $48.1(23.9)$ & $71.8(22.34)$ & 71.17 (19.56) & $\mathrm{EG}(\mathrm{WG}) / \mathrm{BG}$ \\
\hline & & $\mathrm{LF}\left(\ln \mathrm{ms}^{2}\right)$ & $2.9(0.5)$ & $2.5(1.3)$ & $2.59(0.35)$ & $2.83(0.55)$ & - \\
\hline & & $\mathrm{LF} / \mathrm{HF}$ & $4(2.3)$ & $2.6(1.6)$ & $2.81(0.9)$ & $2.91(1.28)$ & - \\
\hline & & SD1 (ms) & $34.8(10.5)$ & 45.7 (15.6) & $34.5(8.16)$ & 35.29 (17.5) & EG (WG) \\
\hline & & SD2 (ms) & $75.5(32.1)$ & $98.21(23.59)$ & $69.3(10.5)$ & 77.57 (12.8) & - \\
\hline \multirow{7}{*}{ Huang (2019) } & \multirow{7}{*}{$\begin{array}{c}10 \text { min } \\
\text { SphygmoCor system } \\
\text { (AtCor Medical, } \\
\text { Sydney, } \\
\text { Australia) }\end{array}$} & SDNN (ms) & $65.2(18.98)$ & $88.3(28.36)$ & - & - & WG \\
\hline & & rMSSD (ms) & $65.8(27.49)$ & $100.5(37.68)$ & - & - & WG \\
\hline & & Pnn50 (\%) & $37.8(17.05)$ & $57.8(12.31)$ & - & - & WG \\
\hline & & $\begin{array}{l}\text { Total Power } \\
\qquad\left(\mathrm{ms}^{2}\right)\end{array}$ & $\begin{array}{c}4012.8 \\
(2272.3)\end{array}$ & $\begin{array}{c}4633.7 \\
(1978.1)\end{array}$ & - & - & - \\
\hline & & $\mathrm{LF}\left(\mathrm{ms}^{2}\right)$ & $\begin{array}{c}1172.8 \\
(1189.5)\end{array}$ & $\begin{array}{l}1882.8 \\
(2465.1)\end{array}$ & - & - & - \\
\hline & & $\mathrm{HF}\left(\mathrm{ms}^{2}\right)$ & $1372.5(908.8)$ & 1988 (983.3) & - & - & - \\
\hline & & $\mathrm{LF} / \mathrm{HF}$ & $1(0.55)$ & $0.7(0.43)$ & - & - & WG \\
\hline \multirow{5}{*}{ Prado (2010) } & \multirow{5}{*}{$\begin{array}{c}3 \mathrm{~min} \\
\text { 12-lead ECG } \\
\text { (Marquette Medical } \\
\text { Systems, CardioSoft, } \\
\text { Wisconsin, USA) }\end{array}$} & $\mathrm{LF}\left(\mathrm{ms}^{2}\right)$ & - & - & - & - & $\mathrm{BG} / \mathrm{EG}(\mathrm{WG})$ \\
\hline & & $\mathrm{HF}\left(\mathrm{ms}^{2}\right)$ & - & - & - & - & $\mathrm{BG} / \mathrm{EG}(\mathrm{WG})$ \\
\hline & & LF (nu) & - & - & - & - & $\mathrm{BG} / \mathrm{EG}(\mathrm{WG})$ \\
\hline & & $\mathrm{HF}$ (nu) & - & - & - & - & $\mathrm{BG} / \mathrm{EG}(\mathrm{WG})$ \\
\hline & & $\mathrm{LF} / \mathrm{HF}$ & - & - & - & - & BG/EG (WG) \\
\hline \multirow{3}{*}{$\begin{array}{l}\text { Vasconcellos } \\
\text { (2015) }\end{array}$} & \multirow{3}{*}{$\begin{array}{c}5 \mathrm{~min} \\
\text { (Polar RS800cx, } \\
\text { Polar }^{\mathrm{TM}}, \text { Kempele, } \\
\text { Finland) }\end{array}$} & LF (nu) & - & - & - & - & WG \\
\hline & & $\mathrm{HF}$ (nu) & - & - & - & - & WG \\
\hline & & $\mathrm{LF} / \mathrm{HF}$ & - & - & - & - & \\
\hline
\end{tabular}

RR, R to R interval; SDNN, standard deviation of all normal-to-normal RR intervals, pNN50, percentage of intervals $>50 \mathrm{~ms}$ different from the previous interval; RMSSD, square root of mean of squares of successive differences of interval RR; LF/HF, low frequency (LF) $\left(\mathrm{ms}^{2}\right) /$ high frequency $(\mathrm{HF})\left(\mathrm{ms}^{2}\right)$ ratio; total power (sum of all spectra); nu, normalized units; In, natural logarithm; SD1, standard deviation of points perpendicular to axis of line of identity in Poincaré plot; SD2, standard deviation of points along axis of line of identity in Poincaré plot; WG, within-group; BG, between-group.

Between-group differences were detected in 7 articles [26,27,30,33-36]. Four of them were randomized controlled trials [26,34-36], $2[27,30]$ were classified as nonrandomized controlled trials (as groups were not randomly allocated), and 1 [33] had a crossover design. Only 1 randomized controlled trial [25] did not report between-group differences (although within-group differences were reported). 


\section{Discussion}

The present systematic review performed up-to-date analysis of studies focused on the effects of physical-exercise interventions on HRV in obese children and adolescents. Physical-exercise interventions mainly focused on aerobic training, but alternative exercise activities, including judo and recreational soccer, were found. The duration of interventions ranged from 6 to 24 weeks with training frequency between 2 and 7 times per week. The duration of sessions typically ranged from 40 to $60 \mathrm{~min}$. From intervention results, HRV increased after physical-exercise interventions in obese children and adolescents, leading to a reduction in sympathetic modulation. However, while 10 articles were included in this systematic review, the heterogeneity of the procedures and methodological concerns may have increased the risk of bias. As such, this interpretation of results must be considered with caution.

Previous studies confirmed that childhood obesity induced abnormal autonomic modulation (presentation of reduced HRV), which is related to poor cardiovascular health [21,22]. This is relevant, as cardiovascular health and, thereby, childhood lifestyle can compromise health in adulthood [37]. In this regard, previous studies indicated that high HRV could be considered a health biomarker [38] for critical illness in children [39]. Thus, considering the effects of physical-exercise interventions on HRV in obese children and adolescents, the autonomic modulation of these populations could be enhanced.

One sustainable development goal is to ensure healthy lives and promote wellbeing at all ages [6]. Considering both the negative impact of obesity on health and the large global prevalence, childhood obesity dramatically affects the achievement of this goal. Of all the negative consequences of obesity, cardiovascular health can be highlighted due to the high health-related costs $[40,41]$ and the relationship between cardiovascular health and mortality $[42,43]$. Thus, physical-exercise interventions that aim to reduce weight and improve cardiovascular health in children and adolescents are crucial to achieving a sustainable future [7-9].

Results of the articles included in this systematic review showed that between-group differences (experimental vs. control) were found in 7 of the 10 articles [26,27,30,33-36]. Among them, 4 were randomized controlled trials [26,34-36] (only 1 randomized controlled trial did not observe between-group differences), $2[27,30]$ were classified as nonrandomized controlled trials (as groups were not randomly allocated), and 1 [33] had a crossover design. These results reinforce the hypothesis of physical exercise's utility as a tool to restore the correct functioning of autonomic modulation in obese children and adolescents. However, future studies should focus on how obesity can impair autonomic modulation. In this regard, obesity disrupts the normal maturation of cardiac autonomic control [44] and would, therefore, favor a reduction in autonomic activity $[45,46]$ or an increase in sympathetic activity $[45,47]$.

One of the main problems in physical-exercise intervention in obese children and adolescents is adherence. Adolescents are not typically motivated by physical-exercise intervention [48], which may lead to ineffective exercise [30]. Taking into account these relevant concerns, some articles included in this systematic review $[25,27,31]$ conducted alternative physical-exercise interventions (compared with traditional aerobic interventions), such as customized training (based on participant interest), judo, or recreational soccer, in order to increase motivation and adherence to programs. These interventions were based on a previous study that indicated that adolescents are more interested in physical activities that are social, outdoors, and competitive. Results from these three interventions demonstrated that judo [27] obtained $74.07 \%$ adherence, recreational soccer [25] 62.5\% adherence, and personalized aerobic training [31] 78\% adherence. However, comparing the adherence of these interventions with the mean adherence of the rest of the program $(86.38 \%)$ reveals that adherence in these three intervention programs was lower. Thus, future randomized controlled trials should address this topic in order to better understand these controversial results. 
Limitations in this systematic review should be noted. First, only articles in English, French, Italian, Portuguese, and Spanish were included. Thus, relevant articles in other languages could have not been found. Second, the heterogeneity of the procedures did not allow for meta-analysis to clarify the extent of HRV improvement after physical-exercise interventions. Third, the results of the systematic review must be considered with caution due to the identified risk of bias sources in the included articles.

\section{Conclusions}

Physical-exercise intervention increased the HRV and thereby improved the autonomic modulation of obese children and adolescents. This is relevant, as HRV is associated with cardiovascular health. The duration of interventions ranged from 6 to 24 weeks, with training frequency between 2 and 7 times per week. The duration of sessions typically ranged from 40 to $60 \mathrm{~min}$. Physical-exercise interventions mainly focused on aerobic training ( $60 \%-75 \%$ of maximal heart rate), but also included alternative interventions such as judo, personalized aerobic training, or recreational soccer. However, extracted evidence from this systematic review did not confirm that these alternative interventions improve adherence to physical exercise.

Author Contributions: Conceptualization, S.V. and D.C.-M.; methodology, D.C.-M., J.L.L.-L. and S.V.; formal analysis, S.V., J.L.L.-L. and J.P.F.-G.; investigation, J.L.L.-L. and S.V.; data curation, J.L.L.-L., J.P.F.-G. and S.V.; writing—original-draft preparation, J.P.F.-G., S.V. and J.L.L.-L.; writing—review and editing, J.P.F.-G. and D.C.-M.; supervision, J.P.F.-G. All authors have read and agreed to the published version of the manuscript.

Funding: This study was made possible thanks to contributions from the Department of Economy and Infrastructure of the Junta de Extremadura through the European Regional Development Fund (GR18129 and GR18155). Author S.V. was supported by a grant from the regional Department of Economy and Infrastructure of the Government of Extremadura and the European Social Fund (PD16008). Author JLLL was supported by a grant from the Spanish Ministry of Education, Culture and Sport (FPU18/05655).

Institutional Review Board Statement: Not applicable.

Informed Consent Statement: Not applicable.

Data Availability Statement: Data will be made available upon reasonable request to the corresponding author.

Conflicts of Interest: The authors declare no conflict of interest.

\section{References}

1. NCD-Risk-Factor-Collaboration. Worldwide trends in body-mass index, underweight, overweight, and obesity from 1975 to 2016 : A pooled analysis of 2416 population-based measurement studies in 128.9 million children, adolescents, and adults. Lancet 2017, 390, 2627-2642. [CrossRef]

2. Jastreboff, A.M.; Kotz, C.M.; Kahan, S.; Kelly, A.S.; Heymsfield, S.B. Obesity as a disease: The obesity society 2018 position statement. Obesity 2019, 27, 7-9. [CrossRef]

3. Reilly, J.J.; Methven, E.; McDowell, Z.C.; Hacking, B.; Alexander, D.; Stewart, L.; Kelnar, C.J.H. Health consequences of obesity. Arch. Dis. Child. 2003, 88, 748-752. [CrossRef] [PubMed]

4. Kamijo, K.; Pontifex, M.B.; O'Leary, K.C.; Scudder, M.R.; Wu, C.T.; Castelli, D.M.; Hillman, C.H. The effects of an afterschool physical activity program on working memory in preadolescent children. Dev. Sci. 2011, 14, 1046-1058. [CrossRef]

5. Pelone, F.; Specchia, M.L.; Veneziano, M.A.; Capizzi, S.; Bucci, S.; Mancuso, A.; Ricciardi, W.; de Belvis, A.G. Economic impact of childhood obesity on health systems: A systematic review. Obes. Rev. 2012, 13, 431-440. [CrossRef] [PubMed]

6. Sachs, J.D. From millennium development goals to sustainable development goals. Lancet 2012, 379, 2206-2211. [CrossRef]

7. Wolters, E.A.; Steel, B.S. Sustainable futures: Healthy lifestyles, obesity, and access to food in US counties 2012. Agric. Agric. Sci. Procedia 2016, 8, 73-80. [CrossRef]

8. Skouteris, H.; Cox, R.; Huang, T.; Rutherford, L.; Edwards, S.; Cutter-Mackenzie, A. Promoting obesity prevention together with environmental sustainability. Health Promot. Int. 2014, 29, 454-462. [CrossRef]

9. The Lancet Diabetes Endocrinology. Obesity and sustainability-in transition. Lancet Diabetes Endocrinol. 2019, 7, 161. [CrossRef]

10. World Health Organization. Global Action Plan on Physical Activity 2018-2030: More Active People for a Healthier World; World Health Organization: Geneva, Switzerland, 2019. 
11. Prado, W.L.; Siegfried, A.; Dâmaso, A.R.; Carnier, J.; Piano, A.D.; Siegfried, W. Effects of long-term multidisciplinary inpatient therapy on body composition of severely obese adolescents. J. Pediatr. 2009, 85, 243-248. [CrossRef]

12. Tjønna, A.E.; Stølen, T.O.; Bye, A.; Volden, M.; Slørdahl, S.A.; Ødegård, R.; Skogvoll, E.; Wisløff, U. Aerobic interval training reduces cardiovascular risk factors more than a multitreatment approach in overweight adolescents. Clin. Sci. 2009, 116, 317-326. [CrossRef]

13. Ingul, C.B.; Tjonna, A.E.; Stolen, T.O.; Stoylen, A.; Wisloff, U. Impaired cardiac function among obese adolescents: Effect of aerobic interval training. Arch. Pediatrics Adolesc. Med. 2010, 164, 852-859. [CrossRef]

14. Balagopal, P.; De Ferranti, S.D.; Cook, S.; Daniels, S.R.; Gidding, S.S.; Hayman, L.L.; McCrindle, B.W.; Mietus-Snyder, M.L.; Steinberger, J. Nontraditional risk factors and biomarkers for cardiovascular disease: Mechanistic, research, and clinical considerations for youth: A scientific statement from the American Heart Association. Circulation 2011, 123, 2749-2769. [CrossRef]

15. Steinberger, J.; Kelly, A.S. Challenges of existing pediatric dyslipidemia guidelines: Call for reappraisal. Circulation 2008, 117, 9-10. [CrossRef] [PubMed]

16. Maggio, A.B.R.; Aggoun, Y.; Martin, X.E.; Marchand, L.M.; Beghetti, M.; Farpour-Lambert, N.J. Long-term follow-up of cardiovascular risk factors after exercise training in obese children. Int. J. Pediatric Obes. 2011, 6, e603-e610. [CrossRef] [PubMed]

17. Guthold, R.; Stevens, G.A.; Riley, L.M.; Bull, F.C. Global trends in insufficient physical activity among adolescents: A pooled analysis of 298 population-based surveys with 1.6 million participants. Lancet Child Adolesc. Health 2020, 4, 23-35. [CrossRef]

18. Freeman, J.V.; Dewey, F.E.; Hadley, D.M.; Myers, J.; Froelicher, V.F. Autonomic nervous system interaction with the cardiovascular system during exercise. Prog. Cardiovasc. Dis. 2006, 48, 342-362. [CrossRef]

19. Dekker, J.M.; Crow, R.S.; Folsom, A.R.; Hannan, P.J.; Liao, D.; Swenne, C.A.; Schouten, E.G. Low heart rate variability in a 2-minute rhythm strip predicts risk of coronary heart disease and mortality from several causes-The ARIC study. Circulation 2000, 102, 1239-1244. [CrossRef] [PubMed]

20. Cohen, M.; Syme, C.; McCrindle, B.W.; Hamilton, J. Autonomic nervous system balance in children and adolescents with craniopharyngioma and hypothalamic obesity. Eur. J. Endocrinol. 2013, 168, 845-852. [CrossRef] [PubMed]

21. Kaufman, C.L.; Kaiser, D.R.; Steinberger, J.; Kelly, A.S.; Dengel, D.R. Relationships of cardiac autonomic function with metabolic abnormalities in childhood obesity. Obesity 2007, 15, 1164-1171. [CrossRef]

22. Rabbia, F.; Silke, B.; Conterno, A.; Grosso, T.; De Vito, B.; Rabbone, I.; Chiandussi, L.; Veglio, F. Assessment of cardiac autonomic modulation during adolescent obesity. Obesity Res. 2003, 11, 541-548. [CrossRef] [PubMed]

23. da Silva, D.F.; Bianchini, J.A.A.; Antonini, V.D.S.; Hermoso, D.A.M.; Lopera, C.A.; Pagan, B.G.M.; McNeil, J.; Junior, N.N. Parasympathetic cardiac activity is associated with cardiorespiratory fitness in overweight and obese adolescents. Pediatric Cardiol. 2014, 35, 684-690. [CrossRef]

24. Amano, M.; Kanda, T.; Ue, H.; Moritani, T. Exercise training and autonomic nervous system activity in obese individuals. Med. Sci. Sports Exerc. 2001, 33, 1287-1291. [CrossRef] [PubMed]

25. Vasconcellos, F.; Seabra, A.; Cunha, F.; Montenegro, R.; Penha, J.; Bouskela, E.; Nogueira Neto, J.F.; Collett-Solberg, P.; Farinatti, P. Health markers in obese adolescents improved by a 12-week recreational soccer program: A randomised controlled trial. J. Sports Sci. 2016, 34, 564-575. [CrossRef] [PubMed]

26. Farah, B.Q.; Ritti-Dias, R.M.; Balagopal, P.; Hill, J.O.; Prado, W.L. Does exercise intensity affect blood pressure and heart rate in obese adolescents? A 6-month multidisciplinary randomized intervention study. Pediatric Obes. 2014, 9, 111-120. [CrossRef] [PubMed]

27. Brasil, I.; Monteiro, W.; Lima, T.; Seabra, A.; Farinatti, P. Effects of judo training upon body composition, autonomic function, and cardiorespiratory fitness in overweight or obese children aged 8 to 13 years. J. Sports Sci. 2020, 38, 2508-2516. [CrossRef] [PubMed]

28. Liberati, A.; Altman, D.G.; Tetzlaff, J.; Mulrow, C.; Gøtzsche, P.C.; Ioannidis, J.P.; Clarke, M.; Devereaux, P.J.; Kleijnen, J.; Moher, D. The PRISMA statement for reporting systematic reviews and meta-analyses of studies that evaluate health care interventions: Explanation and elaboration. PLoS Med. 2009, 6, e1000100. [CrossRef]

29. Kennedy, C.E.; Fonner, V.A.; Armstrong, K.A.; Denison, J.A.; Yeh, P.T.; O'Reilly, K.R.; Sweat, M.D. The evidence project risk of bias tool: Assessing study rigor for both randomized and non-randomized intervention studies. Syst. Rev. 2019, 8, 3. [CrossRef]

30. Farinatti, P.; Neto, S.R.M.; Dias, I.; Cunha, F.A.; Bouskela, E.; Kraemer-Aguiar, L.G. Short-term resistance training attenuates cardiac autonomic dysfunction in obese adolescents. Pediatric Exerc. Sci. 2016, 28, 374-380. [CrossRef]

31. Faulkner, M.S.; Michaliszyn, S.F.; Hepworth, J.T.; Wheeler, M.D. Personalized exercise for adolescents with diabetes or obesity. Biol. Res. Nurs. 2014, 16, 46-54. [CrossRef]

32. Huang, J.; Lai, Q.; Wang, D.; Yin, H.; Liao, J.; Wang, S.; Xu, F.; Hou, X.; Hu, M. Effects Of Exercise Training With Dietary Restriction On Arterial Stiffness, Central Hemodynamic Parameters And Cardiac Autonomic Function In Obese Adolescents. Diabetes Metab. Syndr. Obes. Targets Ther. 2019, 12, 2157. [CrossRef]

33. Gutin, B.; Barbeau, P.; Litaker, M.S.; Ferguson, M.; Owens, S. Heart rate variability in obese children: Relations to total body and visceral adiposity, and changes with physical training and detraining. Obes. Res. 2000, 8, 12-19. [CrossRef] [PubMed]

34. Prado, D.M.; Silva, A.G.; Trombetta, I.C.; Ribeiro, M.M.; Guazzelli, I.C.; Matos, L.N.; Santos, M.S.; Nicolau, C.M.; Negrão, C.E.; Villares, S.M. Exercise training associated with diet improves heart rate recovery and cardiac autonomic nervous system activity in obese children. Int. J. Sports Med. 2010, 31, 860-865. [CrossRef] 
35. Chen, S.-R.; Tseng, C.-L.; Kuo, S.-Y.; Chang, Y.-K. Effects of a physical activity intervention on autonomic and executive functions in obese young adolescents: A randomized controlled trial. Health Psychol. 2016, 35, 1120. [CrossRef]

36. Hamila, A.; Younes, M.; Cottin, F.; Amor, Y.B.; Shephard, R.; Tabka, Z.; Bouhlel, E. Effects of walking exercises on body composition, heart rate variability, and perceptual responses in overweight and obese adolescents. Sci. Sports 2018, 33, e191-e202. [CrossRef]

37. Celermajer, D.S.; Ayer, J.G.J. Childhood risk factors for adult cardiovascular disease and primary prevention in childhood. Heart 2006, 92, 1701-1706. [CrossRef] [PubMed]

38. Young, H.A.; Benton, D. Heart-rate variability: A biomarker to study the influence of nutrition on physiological and psychological health? Behav. Pharmacol. 2018, 29, 140-151. [CrossRef] [PubMed]

39. Marsillio, L.E.; Manghi, T.; Carroll, M.S.; Balmert, L.C.; Wainwright, M.S. Heart rate variability as a marker of recovery from critical illness in children. PLoS ONE 2019, 14, e0215930. [CrossRef] [PubMed]

40. Tarride, J.-E.; Lim, M.; DesMeules, M.; Luo, W.; Burke, N.; O’Reilly, D.; Bowen, J.; Goeree, R. A review of the cost of cardiovascular disease. Can. J. Cardiol. 2009, 25, e195-e202. [CrossRef]

41. Movsisyan, N.K.; Vinciguerra, M.; Medina-Inojosa, J.R.; Lopez-Jimenez, F. Cardiovascular Diseases in Central and Eastern Europe: A Call for More Surveillance and Evidence-Based Health Promotion. Ann. Glob. Health 2020, 86, 21. [CrossRef] [PubMed]

42. Chen, Y.; Freedman, N.D.; Albert, P.S.; Huxley, R.R.; Shiels, M.S.; Withrow, D.R.; Spillane, S.; Powell-Wiley, T.M.; de González, A.B. Association of cardiovascular disease with premature mortality in the United States. JAMA Cardiol. 2019, 4, 1230-1238. [CrossRef] [PubMed]

43. Naghavi, M.; Abajobir, A.A.; Abbafati, C.; Abbas, K.M.; Abd-Allah, F.; Abera, S.F.; Aboyans, V.; Adetokunboh, O.; Afshin, A.; Agrawal, A. Global, regional, and national age-sex specific mortality for 264 causes of death, 1980-2016: A systematic analysis for the Global Burden of Disease Study 2016. Lancet 2017, 390, 1151-1210. [CrossRef]

44. Blüher, S.; Petroff, D.; Keller, A.; Wagner, A.; Classen, J.; Baum, P. Effect of a 1-Year Obesity Intervention (KLAKS Program) on Preexisting Autonomic Nervous Dysfunction in Childhood Obesity. J. Child Neurol. 2015, 30, 1174-1181. [CrossRef]

45. Eyre, E.L.; Duncan, M.J.; Birch, S.L.; Fisher, J.P. The influence of age and weight status on cardiac autonomic control in healthy children: A review. Auton. Neurosci. Basic Clin. 2014, 186, 8-21. [CrossRef] [PubMed]

46. Nagai, N.; Moritani, T. Effect of physical activity on autonomic nervous system function in lean and obese children. Int. J. Obes. 2004, 28, 27-33. [CrossRef] [PubMed]

47. Cote, A.T.; Harris, K.C.; Panagiotopoulos, C.; Sandor, G.G.; Devlin, A.M. Childhood obesity and cardiovascular dysfunction. J. Am. Coll. Cardiol. 2013, 62, 1309-1319. [CrossRef]

48. Krustrup, P.; Aagaard, P.; Nybo, L.; Petersen, J.; Mohr, M.; Bangsbo, J. Recreational football as a health promoting activity: A topical review. Scand. J. Med. Sci. Sports 2010, 20, 1-13. [CrossRef] 